aus als von ihren Fraktionen im Stadtparlament. „Mit dem Generationenwechsel und unter dem Eindruck der allgemeinen 68er-Proteststimmung im Land kam in den Frankfurter Parteien eine Entwicklung in Gang, die das politische Klima in der Stadt grundlegend verändern und 1972 in der Beendigung der jahrzehntelangen Zusammenarbeit von SPD und CDU gipfeln sollte“ (S. 151). Schließlich verweigerte der Parteitag der SPD die Zustimmung zu einer weiteren Zusammenarbeit mit den Christdemokraten und lehnte die Wahl zweier CDU-Dezernenten ab. Das Ende der Koalition wurde vor allem von der Jungen Union innerhalb der CDU an der Spitze als Befreiung empfunden. Die parteipolitische Polarisierung wurde dadurch verschärft, dass es 1977 erstmals zu einem Wechsel in Frankfurt kam und die CDU den Oberbürgermeister stellte. Dennoch band die CDU weiterhin SPD-Dezernenten, wie beispielsweise den Kulturpolitiker Hilmar Hoffmann, in den Magistrat ein. Auch das Parteiensystem geriet in Bewegung, als 1981 die Grünen erstmals in den Römer einzogen und die FPD sechzehn Jahre lang pausieren musste. Tüffers zeichnet den Weg der Parlamentarisierung der Grünen in Frankfurt mit dem Sieg der Realos über die Fundis nach und zeigt in Anlehnung an Joachim Raschke, wie Parlamente als „Konventionsmaschine" wirken (S. 254).

Nachdem Tüffers den dritten Band in der Reihe der Geschichtsschreibung des Frankfurter Stadtparlamentes seit 1867 vorgelegt hat (herausgegeben von der Frankfurter Historischen Kommission), freut man sich schon auf die Fortsetzung bis in die Gegenwart. Einzig störend an der im Grunde klugen und detailreichen Darstellungsweise sind manche übertriebenen eigenen Wertungen der Autorin, beispielsweise wenn sie die durch den Einzug der Grünen ins Parlament veränderte Debattenkultur als „überhand nehmende Monstersitzungen" und als fatale Mischung beschreibt (S. 213). Auch ihre Interpretation, bei der Lektüre eines Protokolls „das kollektive Aufstöhnen der anderen zu hören“ (S. 227), gehört zu den störenden Seitenhieben, die man bei einer Überarbeitung hätte streichen sollen.

Arijana Neumann

\title{
Parteien und Parteiensysteme: vielleicht doch das perfekte Lehrbuch
}

Detterbeck, Klaus: Parteien und Parteiensystem, UVK Verlagsgesellschaft mbH, Konstanz 2011, 272 Seiten, €19,90.

Der Autor wollte eine Einführung in die Parteienforschung schreiben. Vor diesem Hintergrund muss die überschaubare (wenngleich überlange) Gliederung seines Werkes gelobt werden, in die tatsächlich die wichtigsten Felder dieser Forschungsrichtung eingeflossen sind: die Parteienbegriffe, die Binnenorganisation, die Ursprünge, Funktionen, Entwicklungsetappen und Ideologien der Parteien, der elektorale Prozess und die Parteiensysteme in verschiedenen institutionellen Systemzusammenhängen. Klaus Detterbeck veranschaulicht zudem die Hauptaussagen der klassischen Parteienliteratur sowie statistische Daten mittels eigens erstellter Abbildungen und Tabellen - in der Absicht, die auf visuelle Reize stark reagierende Generation der Bachelor- und Masterstudiengänge zu erreichen. Nach dem Vorbild vieler US-amerikanischer Lehrbücher fügt er zudem jedem Kapitel einen Fragenkatalog und auch Links zum Erlernen und Vertiefen des Gelesenen hinzu. 
Ein perfektes Lehrbuch also? Eher ein braves, denn Detterbeck vermittelt lediglich die Namen und Thesen der von jeher als wichtig erkannten Politikwissenschaftler, wobei es ihm „methodisch“ offenbar vor allem daran liegt, seine Überblicksdarstellung mit Seymour M. Lipset, Stein Rokkan sowie Giovanni Sartori zu beginnen und möglichst vollständig wie up to date zu halten. Des Autors eigener theoretischer Zugang zum Gegenstand eröffnet sich dem Leser ebenso wenig wie ein Leitfaden der Darstellung, weshalb sich die auf den ersten Blick überschaubar wirkende Struktur während der Lektüre doch letztlich als zerstückelt erweist. Geradezu beiläufig in einem Satz des Vorworts erhebt Detterbeck den Anspruch, „für die einzelnen Teilgebiete des Themas einen Überblick über die wichtigsten theoretischen Erklärungsmuster" (S. 9) zu bieten, was er (unter Auslassung von jeglichem Theorieverständnis) als „theoriegeleitete“ Vorgehensweise bezeichnet. Im nächsten Satz fügt er noch das Adjektiv „vergleichend“ zur Kennzeichnung seines Buchs hinzu, und zwar mit der Begründung, es hebe die Eigenarten der deutschen Parteien hervor, indem sie in Bezug zu anderen Ländern gestellt würden.

Es scheint ihn weder die Tatsache zu stören, dass es auf Deutsch bereits mehrere auf ähnliche Weise zusammengestellte Bücher gibt, noch verunsichert ihn die Tatsache, dass im englischsprachigen Raum, auf dessen Literatur er sich vorwiegend bezieht, ein Überblick über eine Forschungsrichtung normalerweise in Readern gegeben wird, in die nicht Exzerpte der Dozenten, sondern Originaltextausschnitte gehören. Vor dem Hintergrund der ausbleibenden theoretischen Selbstverortung verwundert zwar nicht, dass Detterbeck zu Kontroversen des Fachs keine Stellung bezieht. Dass sich daraus jedoch ein eher magerer Duktus ergibt, darf auch nicht überraschen. Auf diese Weise entkommt der Autor dem Risiko des Irrtums und des Widerspruchs und erhebt sich zu einem unangreifbaren Alleswisser.

Analytisch kann diese Narration selbstverständlich nicht allzu ergiebig sein, wie hier am Beispiel der Ausführungen über die Wählervolatilität und das Dealignment von Wählern und Parteien veranschaulicht werden kann. Zunächst werden beide Phänomene mittels eines „Blicks in die Empirie“ erkannt (S. 46 ff.). Sodann wird auf mehreren Seiten unkritisch und unter Anwendung von Tabellen und Schaubildern Literatur dazu referiert. In einem späteren Unterkapitel wird diese „Empirie“ wiederum zu „Symptomen“ einer verwandten Erscheinung umfunktioniert: „Die Grundlagen des Wahlverhaltens haben sich in den letzten Jahren grundlegend verändert - dies ist die Quintessenz der Wahlforschung in den westlichen Demokratien. Die steigende Volatilität und das Schwinden von Parteibindungen sind deutliche Symptome dieses Wandels. (S. 75) Abgeschlossen wird dieser Abschnitt nach acht Seiten eines mit Schaubildern und Tabellen bestückten Referats über mehrere Autoren mit dem folgenden Fazit: „Der generelle Befund der Wahlforschung, dass sich die Bindungen zwischen Parteien und Wählern gelockert haben, bleibt ... unangetastet. Der Wahlmarkt ist fluider und volatiler geworden“ (S. 82). Die Sprunghaftigkeit und Loslösung der Wähler von Parteibindungen werden also einmal als das zu erklärende Phänomen, einmal als der Indikator verwandter Phänomene und dann noch als „der generelle Befund“ beziehungsweise „die Quintessenz der Wahlforschung" verstanden. Schade, dass der Autor die Frage nicht stellt, wozu denn aber angesichts solcher Befunde die Parteienforschung gut sein sollte? Diese Erscheinungen sind doch jedem bekannt, der sich ab und zu Wahlergebnisse anschaut.

Das Buch fügt sich einwandfrei in jene Tradition der deutschen Politikwissenschaft, die man „hegelianisch“ nennen kann, weil für sie Ideen - im Sinne breit begriffener Theorien - wichtiger als die zu erklärende Wirklichkeit sind (was dazu führt, dass die Aussagen der 
Theorien gern mit der Wirklichkeit selbst verwechselt werden). In diesem Sinne hat sich der Überblicksradius der Parteienforschung seit 1989 für Detterbeck kaum verändert. Europa endet in diesem Buch offenbar auch heute im Bayerischen Wald und in Österreich. Verglichen mit der geographischen Wahrnehmungsgrenze der bundesdeutschen Parteienforschung des Jahres 1989 wagt Detterbeck immerhin eine Neuerung: Die Elbe weicht im Lehrbuch der Oder. Selbst wenn das Auslassen der Einschränkung „in westlichen Demokratien“ im Buchtitel lediglich einen Flüchtigkeitsfehler darstellen sollte, hätten im Text zumindest einige Parteien und Parteiensysteme jener ehemals kommunistisch regierten Länder Erwähnung finden müssen, die seit mehreren Jahren EU-Mitglieder sind. Recht viel könnte man daraus etwa über solche klassischen Themen der komparativen Parteienforschung lernen wie Cleavages, Entstehung und Organisation von Parteien sowie institutionelle Stabilität junger Parteiensysteme.

Selbst der bescheidene Anspruch aus dem Vorwort, einen Überblick über „die Parteienforschung" zu bieten, wird auch deshalb nicht eingelöst, weil Parteien in autoritären Systemen darin nicht einmal angesprochen werden. Unerwähnt bleiben sie ungeachtet der systemischen „Vorherrschaft“ des Autoritarismus in der gegenwärtigen Welt, der zunehmenden Bedeutung der Parteien in diesem Herrschaftssystem und des durchaus ausgiebigen Forschungsstandes.

Man könnte abschließend auch mit Hinblick auf die Zielgruppe des Lehrbuchs beklagen, dass sich Detterbeck ausschließlich an harmoniesüchtige Adepten des Fachs wendet beziehungsweise solche zu erziehen trachtet. Diese Kritik wäre aber nur dann gerecht, wenn in der deutschen Politikwissenschaft die besagte hegelianische Tradition nicht seit Jahrzehnten vorherrschend und die hier skizzierte Narration selbst für wissenschaftliche Qualifizierungsarbeiten nicht längst typisch geworden wäre. Also doch das perfekte Lehrbuch!

Jerzy Maćków

\section{Parteientheorie und direkte Demokratie: gelungene Verknüpfung, übertriebene Einordnung der Befunde}

Hornig, Eike-Christian: Die Parteiendominanz direkter Demokratie in Westeuropa, Nomos Verlagsgesellschaft, Baden-Baden 2011, 344 Seiten, € 59,-.

Eike-Christian Hornigs Grundidee ist es zwei Theorien zu verbinden, nämlich jene der direkten Demokratie und die Parteientheorie. Bei ersterer geht der Verfasser von Sabine Jungs „Logik der direkten Demokratie“ (2001) aus, für letztere knüpft er an den Parteiendominanzansatz von Richard S. Katz und Rudolf Wildenmann in der Weiterentwicklung durch Heidrun Abromeit an. Jungs Frage nach der Kompatibilität von direktdemokratischen Verfahren und repräsentativen Systemen bezieht Hornig also auf die Rolle der Parteien: Werden diese durch direkte Demokratie geschwächt, wie es lange ausgemacht schien, oder gilt umgekehrt, dass die Parteien die direkte Demokratie als neue Arena benutzen und dadurch an Stärke gewinnen, wie es in neueren Arbeiten vertreten wird? Seine Grundannahme, dass „Parteien auch die direktdemokratischen Prozesse in ,ihrem“ jeweiligen politischen System dominieren“ (S. 28) will Hornig für Dänemark, Frankreich, Großbritannien, die Nieder- 